\title{
Cascaded Constrained Optimization For Cheetah-Inspired Galloping Robot Leg Mechanism
}

\author{
Artem A. Egorov* Ivan I. Borisov*,** Sergey A. Kolyubin* \\ Stefano Stramigioli ${ }^{*, * * *}$ \\ * Biomechatronics and Energy-Efficient Robotics Lab, ITMO \\ University, St. Petersburg, Russia \\ e-mail: \{aaegorov, borisovii, s.kolyubin\}@itmo.ru \\ ** Center for Technologies in Robotics and Mechatronics Components, \\ Innopolis University, Innopolis, Russia \\ *** Department of Electrical Engineering, Mathematics and Computer \\ Science, University of Twente, The Netherlands
}

\begin{abstract}
The paper describes one of the key steps in creating a bio-inspired galloping robot, namely parametric optimization of a multi-link leg mechanism. We introduce the cascaded constrained optimization procedure for finding optimal values for geometric parameters of a femur, knee, and foot segments for given design constraints and various gait specifications. The presented approach is computationally efficient and guarantees convergence to the unique solution. Steps on free parameters, constraints, and cost function selection are discussed. The efficiency of the proposed procedure is illustrated via a series of simulations. The proposed approach can also be implemented for the design of wearable robots like upper- and lower-limb exoskeletons and rehabilitation systems that should follow sometimes sophisticated human-like trajectories.
\end{abstract}

Copyright $(0) 2020$ The Authors. This is an open access article under the CC BY-NC-ND license (http://creativecommons.org/licenses/by-nc-nd/4.0)

Keywords: Mobile robots, robot design, legged robots, parametric optimization.

\section{INTRODUCTION AND MOTIVATION}

One of the most significant challenges in robotics is creating an energy-efficient legged robot, which can produce stable dynamic locomotion during physical interaction with the ground. The most remarkable advantage of legged machines over wheeled vehicles is the ability to reconfigure and exploit discrete interactions in a large workspace (Kim et al. (2017)). The ability to make and break contacts is essential to move over uneven terrain. During the past decade, a significant number of legged robots have been created: walking, hopping, running, galloping robots with one, two, and multiple legs. All the robots which are mentioned in the paper differ in terms of legs structures, actuation, energy sources, and energy-efficiency.

Concerning the leg structure, several types of mechanisms are used. The most common are the stiff fully actuated open-chain mechanisms (Hutter et al. (2017)) and underactuated mechanisms with the closed loops and elastic elements (Seok et al. (2014)). Leg mechanisms must be adaptive, i.e. passive for a stable physical interaction. The passivity can be achieved whether control algorithms for stiff and rigid systems or via embedding elastic elements into leg mechanism to absorb impact force. Mostly sophisticated impedance control methods are used that demands a custom made actuators (Bledt et al. (2018)) and precise sensors that result in a complex system.

* This work is supported by the Russian Science Foundation grant (project №17-79-20341).
The alternative way is the morphological computation, which results in using inherent robot dynamics and little control efforts to excite, stabilize, or augment the intrinsic dynamics of the mechanical system and the controller shape a robot motion (Folkertsma et al. (2015)). Here we consider the resonance-based approach toward energyefficiency of legged robots, which is one of the most important criteria for legged locomotion. Locomotion could benefit from self-stabilizing properties of the compliant robot design (Spröwitz et al. (2013)), and it matches our desire to develop an intelligent physical design.

This paper focuses on the leg mechanism synthesis for a galloping robot, which uses a simple position and velocity control to induce dynamic locomotion based on a resonance of an elastic element embedded into the mechanical structure. It must be indicated that the laws of energy are related to the kinematics and dynamics of the system. In this article, we are interested in the structure of the leg, which is mainly associated with kinematics. This paper is a starting point for further mechanism optimization, including elastic elements allocation and stiffness adjustment (Borisov et al. (2019)). This paper is inspired by the robotic cheetah project at the University of Twente (Snippe (2017); Folkertsma et al. (2015)).

Mechanism synthesis is a crucial procedure during the development of a robotic system since desired motion a specific mechanism is a result of the identification of appropriate dimensions of the linkages. There multiple methods for structural and dimensional synthesis. For 


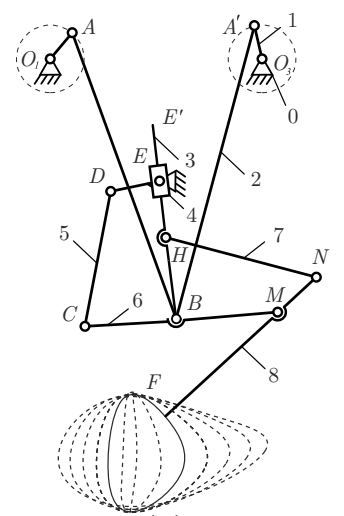

(a)

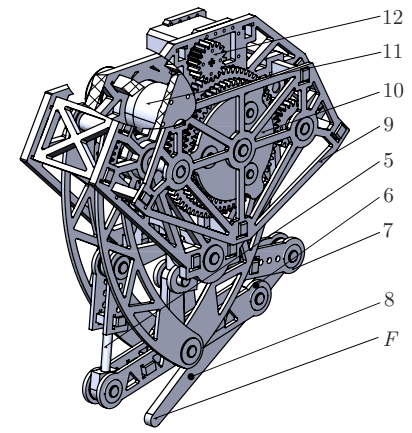

(b)
Fig. 1. The mechanism of the leg along with their change in trajectory $(a)$ and the CAD model of the leg $(b)$

example, Xi et al. (2010) discusses mechanism synthesis for a robot under the condition of minimum acceleration and speed limits of an end-effector. Lum et al. (2006) focuses on the synthesis of a surgical robot, taking into account an instrument working area. Various methods have been developed and applied for legged robots in particular. Ogura et al. (2006) describes the development of a bilateral walking robot with flexible passive legs, where each joint has been optimized to ensure smooth movements similar to a person's gait. He and Geng (2011) tell about dynamic synthesis for a jumping robot to reduce the actuator torque. The Disney-Research team has done a lot for mechanism optimization: for animation tasks (Coros et al. (2013)), jumping and walking robots Megaro et al. (2017), mechanism optimization (Ha et al. (2016)).

This paper describes a cascaded constrained optimization procedure and steps related to cost function, active constraints, and free parameters selection. The suggested approach deals with leg mechanism separation into related subsystems: for the femur, knee, and foot (Fig. 3). Kinematic parameters optimization is carried out consequently for each subsystem. It allows the use of a simpler cost function and less stringent criteria, as well as operating with fewer decision variables. As a result, we uniquely define a mechanism geometry such that it also fits imposed design constraints related to future use of the leg for a complete quadruped assembly.

We should mention that even though this case study is related to legged robot design, the proposed approach is quite general to be applied for mechanism optimization of universal or tailor-made wearable and rehabilitation robotic systems, which satisfy specific ergonomic requirements and can support natural human motions.

The rest of the paper is organized in the following way. Section 2 sets the problem putting requirements for the leg design. The mechanical structure is also described here. Section 3 presents the framework of the proposed cascaded parametric optimization approach. Section 4 describes the solution to the optimization problem with the solid mathematical formulation and its implementation remarks. Section 5 contains the results of the optimization and confirmation of the effectiveness of the methodology. Finally, Section 6 is for conclusions and setting-up directions for future work.

\section{MECHANISM STRUCTURE AND PROBLEM FORMULATION}

The leg mechanical structure is inspired by cheetah anatomy. Since the idea is to reproduce not just appearance but the functionality as well, the mechanism has to copy the feet trajectory of a real animal. The cheetah can move with different gaits, and functions of the front are rear legs differ. Rear legs are more muscular than the front and mainly responsible for the pushing movement. This paper focuses on the rear leg mechanism, which is shown in Fig. 1, a. The structure is discussed in Borisov et al. (2019). The optimization task for kinematic parameters was solved under the assumption that the structure is predetermined and does not change.

\subsection{Mechanism}

The rear leg mechanism is depicted in Fig. 1, $a$. It consists of several links: (1) cranks $O_{1} A$ and $O_{3} A^{\prime}$ which are the input links, (2) connecting rods $A B$ and $A^{\prime} B$, (3) crank arm $B E^{\prime}$, (4) brick $E$, (5) sartorius $D C$, (6) tibia $C B M$, (7) fibula $H N,(8)$ metatarsal $N M F,(0)$ frame, and $F$ is a contact point with the ground. The femur mechanism $O_{1} A B A^{\prime} O_{3}$ is responsible for moving the patella $B$ (Fig. 1, a). The knee mechanism EDCB controls the angle between the femur and the tibia in the specified range. The knee mechanism is attached to the $B$ and the brick $E$. The group of tibia, fibula, and tarsal $H N F M B$ executes pantograph functions to scale point $B$ trajectory. The mechanism has 2 active degrees of freedom and it can produce a large range of different gaits via controlling the phase difference between the cranks. When the phase equals zero, the contact point $F$ travels vertically, but if there is a phase difference, the trajectory becomes ellipsoid-like. The vertical and horizontal parts of the trajectory are uncoupled. The CAD model of the designed leg mechanism is depicted in Fig. 1, $b$. All heavy objects as the primary DC motor with gearbox (11) to actuated both cranks, precision servo motor (12) to control the angle phase between the cranks, and planetary gearbox (10) are installed in robot's body (9). The dynamic behavior can be described using spring loaded inverted pendulum model. Due to the model, all the mass and inertia must be allocated in the robot body, while the leg mechanism is inertialess. Thus, it is reasonable to focus on kinematic issues only on this stage.

\subsection{Problem}

The mechanism can be described as a function transforming the motion of input links to the motion of output point $F$, where links lengths are parameters to optimize. The space of all possible output values can be narrowed due to the function, depending on mechanism constraints. The task is to find optimal free parameters values such that for possible input links' movements and under imposed design constraints, output links follow desired trajectories. It is needed to determine fixed and free parameters, how the desired motion of output links can be parametrized, and what cost function is better in terms of convergence and computational efficiency. Essential requirements for trajectories are convexity at the points of landing and push and the absence of self-intersection. 


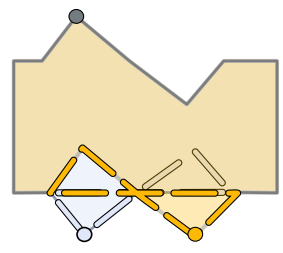

(a)

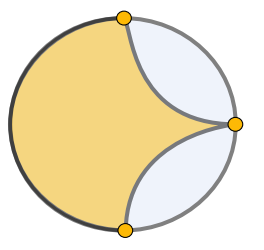

(b) $(c)$

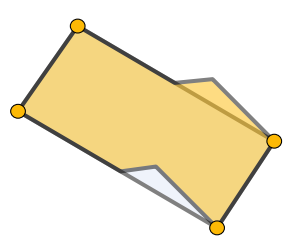

Fig. 2. Examples of shapes with different areas, perimeters and special points position: $(a)$ equal areas and perimeters, but objects are not the same, $(b)$ equal special points and perimeters, but areas differ, $(c)$ equal areas and special points, but perimeters differ

\section{BASIC APPROACH}

A mechanism synthesis includes structure and parametric optimization, considering the desired motion, requirements, and limitations. This task, especially for a multilink mechanism, with analytic tools, is a rather laborious process. Therefore numeric optimization approaches can be a useful tool worth developing.

Since the rear leg mechanism has many parameters to optimize, it worth dividing it onto subsystems. For each of them to define constraints and desired behavior and split optimization procedure into stages. If the problem statement does not give a unique solution, additional optimization constraints should be introduced either as hard constraints or by modifying the cost function.

\subsection{Metrics for Cost Function}

The task is to find optimal kinematic parameters for the mechanism to ensure the output link desired trajectory, velocity profiles, and energy characteristics of the mechanism. A specific metric is needed to measure the quality of solutions. There are two fundamentally different principles for calculating metrics. One way to optimize the kinematic parameters is to minimize the Euclidean distances between corresponding points of desired and current trajectory for the current kinematic parameters. Better accuracy needs an increased number of points, which means that the method requires massive computations. The alternative way is calculating perimeters and areas of the cyclic paths to compare the current trajectory with the desired ones.

However, only these two metrics are not entirely suitable for the considered case. Using it for the leg mechanism optimization cannot guarantee that the desired trajectory height and length to be achieved at the same time, i.e., matching in paths' perimeters and areas does not mean that its shapes are close to each other (examples on Fig. 2). Therefore, we suggest combining within the cost function derivations of perimeters and areas together with the calculating distance between just a few characteristic points of the desired and calculated trajectories (Fig. 4).

\subsection{Cascade}

The proposed metric does not guarantee the correctness of the solution in the general case. It all depends on the number of unknown parameters. Therefore, it is crucial to reduce their number.

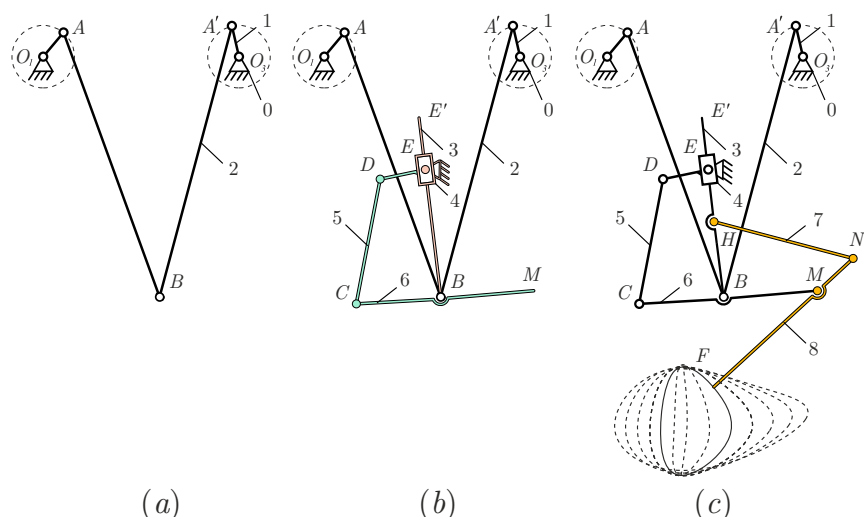

Fig. 3. Leg mechanism decomposition: (a) femur, (b) knee, and $(c)$ foot structures

One way to do it is to divide the task of the full leg synthesize into sub-tasks of optimizing its parts. In order to do it, the leg mechanism was divided into three parts: the femur, the knee, and the foot (Fig. 3). The optimization is organized as a cascade of three sequential steps, where results of the previous stage are used for the next one. Each step has its own free parameters and an output point located at a specific link: $B$ - for the femur, $M$ - for the knee, and $F$ - for the foot.

\section{PARAMETRIC OPTIMIZATION}

\subsection{Optimization constraints}

The femur subsystem. Before the optimization, fixed, desired, and free parameters have to be considered. The following parameters characterize the femur mechanism (Fig. 3, a): four links' lengths and the phase difference between the cranks (1). The crank length is considered as a fixed parameter. It is supposed to be as small and light as possible to decrease inertia and to be fitted in the robot body. The existence conditions of the femur mechanism are:

$$
L_{O_{1} A}+L_{A B}+L_{O_{3} A^{\prime}}+L_{A^{\prime} B} \leq L_{O_{1} O_{3}},
$$

where $L_{O_{3} A^{\prime}}=L_{O_{1} A}, L_{A^{\prime} B}=L_{A B}$, and distance between the ranks' frames $L_{O_{1} O_{3}}$ is fixed since it is parameter was calculated for a planetary gearbox.

It is needed to describe boundaries for the maximum length links due to desired robots dimensions:

$$
\forall L<L_{b p}
$$

where $L_{b p}$ is the maximum length for all links, which is caused by desired dimensions and physical material properties as density, Young's module, Poisson coefficient for the given material. All the links a supposed to be laser-cut from polyoxymethylene to ensure endurance and little inertia. $A B$ and $A^{\prime} B$ were chosen as parameters for optimization.

The limitations of the maximum phase between the input cranks are also taken into account. This allows us to reduce the stroke and dimensions of the parts inside the gearbox, which again makes our design more compact and lighter. After analysis, the mirroring of the output trajectories was found for phases from $0^{\circ}$ to $180^{\circ}$ and from $180^{\circ}$ to $360^{\circ}$, therefore, finally we get:

$$
0^{\circ}<\beta<180^{\circ} \text {. }
$$




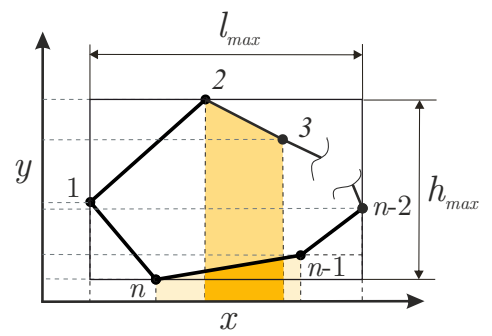

$(a)$

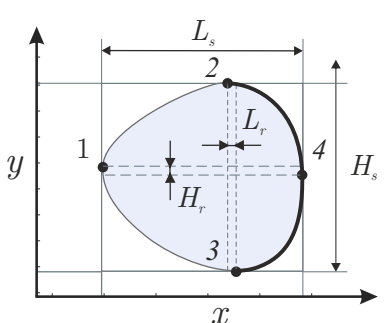

(b)
Fig. 4. (a) polygon constructed out of an array of coordinates, (b) shape of a trajectory, where 1 and 4 are boundaries for the horizontal component and 2 and 3 are for the vertical component of the trajectory respectively, $L_{s}$ - stride length, $H_{s}$ - height

The knee subsystem consists of two Assur groups 3-4 and 5-6. The Assur group is the assembly of links with zero degree of freedom. Using Chebychev-Grübler criterion it can be seen that for the two links with 3 planar joints

$$
W=3 n-2 P_{5}=3 \cdot 2-2 \cdot 3=0,
$$

where $W$ - is the degree of freedom, $n$ - the number of movable links, $P_{5}$ - the number of planar joints with 5 constraints. Brick $E$ and links $D E$ are rigidly connected. The knee subsystem has a greater number of geometric parameters. The optimization can be implemented for any set of parameters. It is considered to choose $D C$ and $C B$ as variables.

The following inequalities are obtained from the conditions of existence for the linkage:

$$
\begin{gathered}
L_{D C}+L_{C B} \geq \sqrt{L_{D E}^{2}+L_{E B \max }^{2}}, \\
L_{H B}<L_{E B \min },
\end{gathered}
$$

where $L_{E B \max }$ and $L_{E B \min }$ are boundaries for links' dimensions to be calculated after optimizing parameters of the femur subsystem. $L_{H B}$ is considered as fixed.

The foot subsystem. The tibia is considered fixed for physical reasons, the fibula is calculated from the geometry, and $M N$ is fixed in consideration of the robot's design. The output link $M F$ should be additionally made in the form of an elastic element. Therefore its length is left as a dynamic parameter. Instead of simulating the whole robot and the full physical model, some constants were introduced. This led to an acceleration of the optimization process with minimal losses in realism.

Cost function. The path traced by the point $F$ is approximated as a shape, specified by planar coordinates of the vertices. The area and perimeter of the shape are calculated by splitting it into segments, which are defined based on the polygon vertices locations (Fig. 4). Shape area can be calculated as follows:

$$
S=\sum_{i=1}^{N-1}\left(S_{i, i+1}\right)+S_{N, 1},
$$

where $S_{i, j}=\frac{\left(x_{j}-x_{i}\right) \cdot\left(y_{j}+y_{i}\right)}{2}$ is the area of the trapezoid with indexes $i$ and $j$ denote respective points numbering. Note that by adding such expressions, we get the area bounded by the spline. It happens because one part of the trapezoid has a positive area, and another is negative (see Fig. 4).

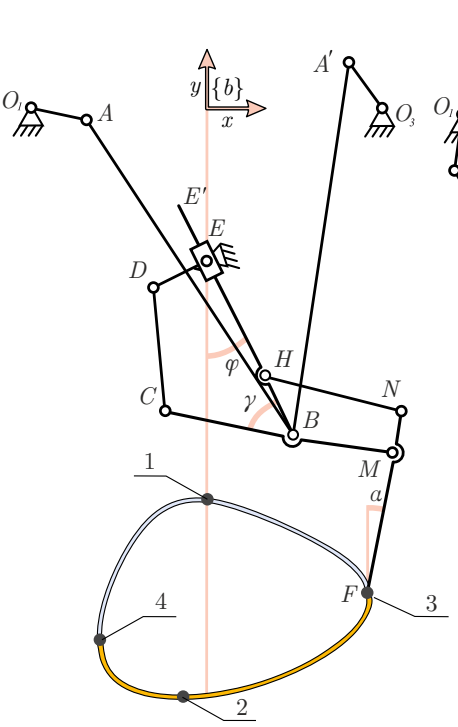

(a)

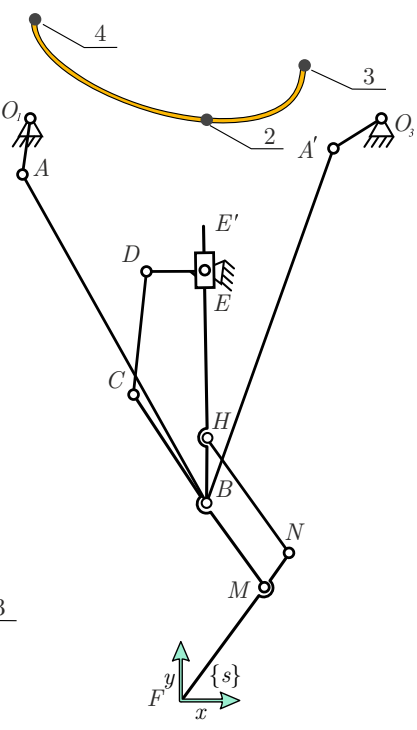

(b)
Fig. 5. Trajectories $(a)$ of point $F$ in body frame $\{b\},(a)$ body in space frame $\{s\}$. 1 - apex, 2 - touchdown, 3 and 4 - boundaries for horizontal component; $\gamma$ is the angle between $B C$ and $H B, \phi$ is the angle between $H B$ and vertical line, $\alpha$ is the pitch angle between $M F$ and vertical.

Polygon perimeter can be calculated as a sum of segment lengths:

$$
P=\sum_{i=2}^{N}\left(L_{i-1, i}\right)+L_{N, 1},
$$

where $L_{j, i}=\sqrt{\left(\left(x_{i}-x_{j}\right)^{2}+\left(y_{i}-y_{j}\right)^{2}\right)}$.

So, the natural choice of the optimization cost function is

$$
F_{1}=\left(S^{*}-S\right)^{2}+\left(P^{*}-P\right)^{2},
$$

where $S^{*}$ and $P^{*}$ denote area and perimeter of the shape related to the desired trajectory, respectively. Therefore, these points are taken from general considerations and experimentally detailed quantities (see Fig. 5). These moments break the trajectory into the phases of stance, takeoff, flight, retraction just before contact, and touchdown.

The updated optimization cost function is

$$
F_{2}=\left(S^{*}-S\right)^{2}+\left(P^{*}-P\right)^{2}+\sum_{i=1}^{4}\left(d_{i}^{2}\right),
$$

where $d_{i}^{2}=\left(x_{i}^{*}-x_{i}\right)^{2}+\left(y_{i}^{*}-y_{i}\right)^{2}$ (Fig. 5). The algorithm has to find the lowest value of the cost function. The optimal solution is the closest to zero. Coincidences of areas and special points have different weights.

Therefore, it is useful to introduce coefficients to regulate their relationship. Thus, another cost function is introduced:

$$
F_{3}=k_{1}\left(S^{*}-S\right)^{2}+k_{2}\left(P^{*}-P\right)^{2}+k_{3} \sum_{i=1}^{4}\left(d_{i}^{2}\right),
$$

where $k_{1}, k_{2}, k_{3}>0$ are weighting coefficients. For example, increasing $k_{3}$ relative to $k_{1}$ and $k_{2}$ we increase the sensitivity of the cost function to deviations in characteristic points displacements. 


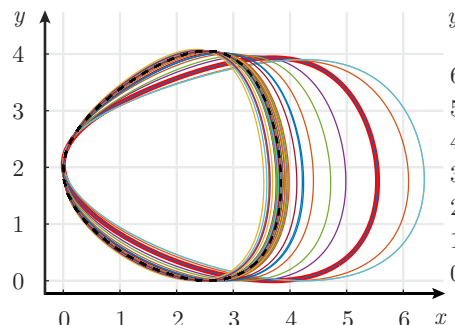

(a)
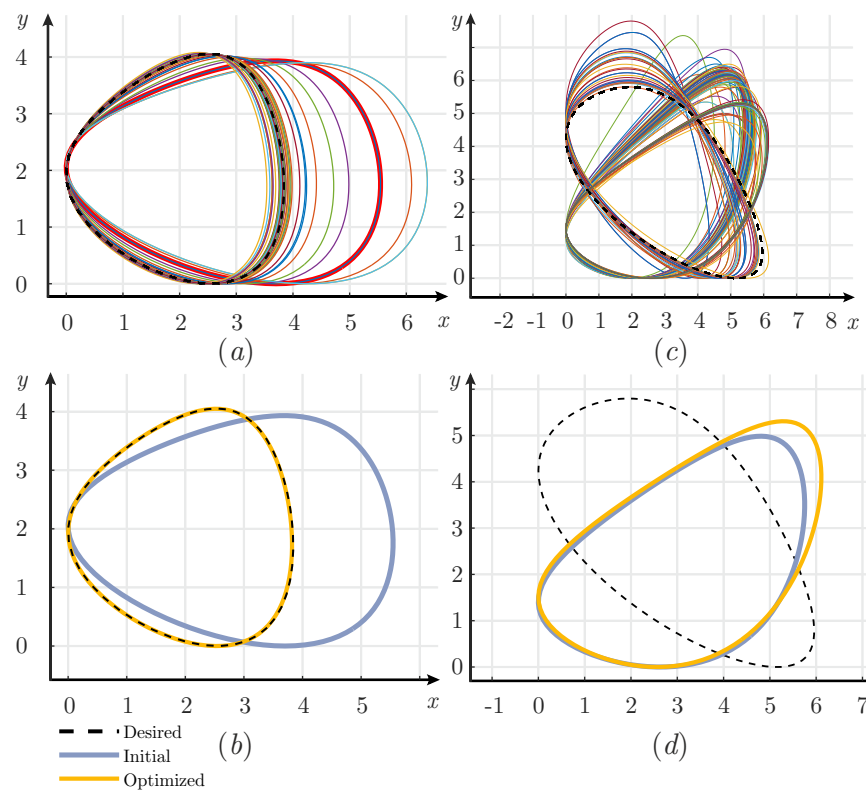

(c)

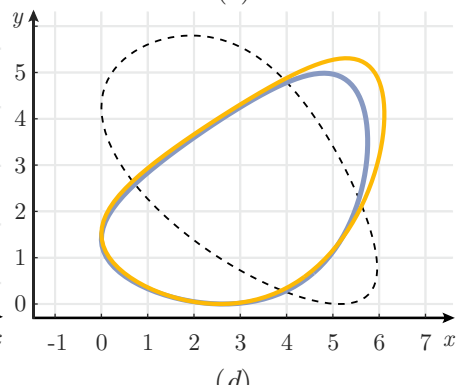

(d)

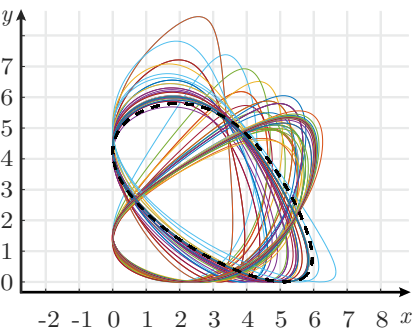

(e)

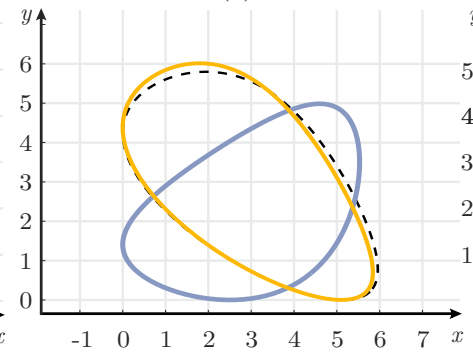

$(f)$

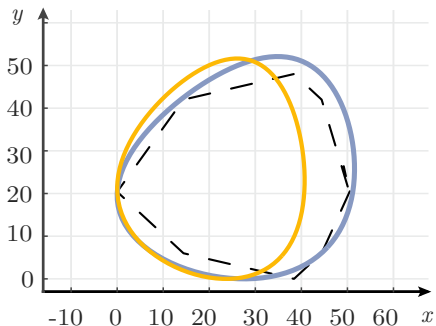

$(g)$

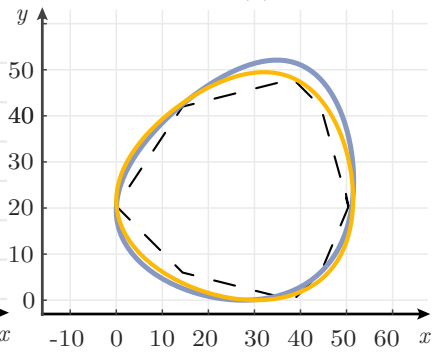

(h)

Fig. 6. The initial trajectories are the bold blue lines; the desired are black dotted lines, and the optimized are the orange. Femur trajectory optimization progress $(a)$ and results $(b)$; all possible knee trajectories in optimization progress with cost function $F_{1}(c)$ and results $(d)$; all possible knee trajectories with cost function $F_{2}(e)$ and results $(f)$; foot trajectory after optimization by cost function $F_{2}(g)$ and $F_{3}$ with the following coefficients $k_{1}=1, k_{2}=1, k_{3}=40000$.

\subsection{Optimization Algorithm}

To find the optimal value of the cost function, the algorithm for constraint nonlinear optimization patternsearch was used (Conn et al. (1991)). It's based on the direct search to solve optimization problems that do not require any information about the gradient of the objective function. Unlike more traditional optimization methods that use information about the gradient or higher derivatives to search for an optimal point, a direct search algorithm searches a set of points around the current point, looking for one where the value of the objective function is lower than the value at the current point. Thus, it can be used to solve problems for which the objective function is not differentiable, or is not even continuous.

\subsection{Geometry}

Not all lengths in a mechanism are optimized or mechanically constant. The length of the fibula is calculated after optimization of the knee from additional physical conditions: pitch angle and finding the moment of contact between the foot and the surface. This subsection is about geometry equations.

Table 1. Optimized mechanism parameters

\begin{tabular}{ccccc} 
Fixed & Value $[\mathrm{mm}]$ & Free & Initial & Optim. [mm $]^{*}$ \\
\hline \hline$O_{1} O_{3}$ & 144 & $\mathrm{AB}$ & 120 & 214.50 \\
$O_{1} E_{x}$ & 72 & $D C$ & 0 & 77.28 \\
$O_{1} E_{y}$ & 100 & $C B$ & 0 & 98.77 \\
$F M_{1}$ & 74 & $M F$ & 0 & 53.18 \\
$M N$ & 31 & $N H$ & 0 & 89.52 \\
$A O$ & 15 & $\beta$ & $20^{\circ}$ & $31.90^{\circ}$ \\
$E D$ & 35 & & & \\
$\mathrm{HB}$ & 35 & & & \\
\hline
\end{tabular}

The length of the $E B$ depends on the mechanism's configuration. At the first stage of optimization, the maximum value was found to calculate the conditions of existence for the linkage $E D C B$. In principle, there are two stages: the search for the value of $\gamma$ and the fibula's length calculation. $C C_{1}$ is perpendicular from $C$ to $E B . D P$ is perpendicular to the $C C_{1}$ from $D$ (Fig. 5):

$$
\begin{gathered}
b=B C, a=D C, l_{1}=D E, l_{2}=E B, \\
C C_{1}=b \cdot \sin (\gamma), B C_{1}=b \cdot \cos (\gamma), \\
C P=C C_{1}-l_{1}, D P=l_{2}-B C_{1}, \\
a^{2}=\left(b \cdot \sin (\gamma)-l_{1}\right)^{2}+\left(l_{2}-b \cdot \cos (\gamma)\right)^{2} .
\end{gathered}
$$

After opening the brackets and transformations, we get:

$$
\begin{gathered}
c=\frac{\left(-b^{2}+l_{1}^{2}+l_{2}^{2}+a^{2}\right)}{2 \cdot a} \\
\tan \left(\frac{\gamma}{2}\right)=\frac{l_{2}}{\left(l_{2}-c\right)} \pm \sqrt{\frac{l_{2}^{2}}{\left(l_{2}-c\right)^{2}}+\frac{l_{1}-c}{l_{2}-c}}
\end{gathered}
$$

From the last equation, we can find out the value of the $\gamma$ angle by choosing plus, because we need a positive angle with respect to $B E$. Knowing the coordinates of points $E$ and $B$, we can calculate the angle $\phi$ from a right triangle.

$$
\angle H B M=180^{\circ}-\gamma, \omega=\angle B H M+\phi
$$

$$
H M^{2}=H B^{2}+B M^{2}-2 \cdot H B \cdot B M \cos \left(180^{\circ}-\gamma\right)
$$

Through the sine theorem, the $\angle B H M$ and $\omega$ as $\angle B H M$ $+\phi$ can be found. $H N$ (fibula) can be calculated using the cosine theorem because $M N$ is defined in advance. The $\angle H M N$ is equal to the sum of $\omega_{1}$ and $\alpha_{1}$, which is equal to $\omega$ and $\alpha$ respectively (as the angles between the secant and parallel lines). 


\section{SIMULATION RESULTS}

The simulation was performed in MATLAB using patternsearch for finding minimum of the cost function. Table 1 shows all the values of the parameters of the final mechanism. Figure $6, a$ shows all possible trajectories in optimizing the femur by cost function $F_{1}$. Figure $6, b$ clearly demonstrates the difference between the desired, optimized and initial results. The following figures demonstrate the unreliability of cost function $F_{1}(9)$ for the knee optimization step (see Fig. $6 c$ and Fig. $6, d$ ), while the function $F_{2}(10)$ gives the correct result (see Fig. 6, $e$ and Fig. $6, f)$. For the last optimization step, the cost function $F_{2}$ (10) was used only for 8 points for calculating areas and perimeters when at the previous stages there were 50 of them(see Fig. $6, g$ ). The cost function $F_{3}(11)$ was able to solve with all the complications(Fig. $6, h$ ).

\section{CONCLUSIONS}

This article presents the algorithm that can be used as a part of a bio-inspired galloping robot leg mechanism design. Since the stucture was predetermined, the main task was to optimize the kinematic parameters of the multibody closed-chain mechanism such that the foot trajectory mimics the cheetah animal behavior. To do that, we had to organize the procedure of nonlinear constrained optimization. Parametric optimization was done in cascade, while we split the entire leg mechanism to 3 subsystems: the femur, the knee, and the foot structures. On the one hand, it allows reducing the number of free parameters and constraints to handle at each optimization step. On the other hand, we can control not only the end-point trajectory but also trajectories of other points of interest like femur and knee joints. The mechanism is optimized for the specific gait; however, via the phase difference control, it is possible to change the gaits (see Borisov et al. (2019)). Practical remarks on a selection of the cost function, free, fixed parameters, and optimization constraints are given, taking into consideration mechanism compactness and light-weight requirements, as well as material strength and manufacturing technologies properties. The advantage of the proposed method is that we can pre-determine the desired behavior of the robotic systems by means of its design optimization that simplifies requirements for further motion control algorithms synthesis and required sensory equipment. This approach also can be used for parametric optimization of a wider class of mechanisms, e.g., upperand lower-limb exoskeletons and robotic rehabilitation systems that should follow human-like trajectories and satisfy ergonomic requirements.

\section{REFERENCES}

Bledt, G., Powell, M.J., Katz, B., Di Carlo, J., Wensing, P.M., and Kim, S. (2018). Mit cheetah 3: Design and control of a robust, dynamic quadruped robot. In 2018 IEEE/RSJ International Conference on Intelligent Robots and Systems (IROS), 2245-2252. IEEE.

Borisov, I.I., Kulagin, I.A., Larkina, A.E., Egorov, A.A., Kolyubin, S.A., and Stramigioli, S. (2019). Study on elastic elements allocation for energy-efficient robotic cheetah leg. In 2019 IEEE/RSJ International Conference on Intelligent Robots and Systems (IROS), 16961701. IEEE.
Conn, A.R., Gould, N.I.M., and Toint, P. (1991). A globally convergent augmented lagrangian algorithm for optimization with general constraints and simple bounds. SIAM Journal on Numerical Analysis, 28(2), 545-572. doi:10.1137/0728030.

Coros, S., Thomaszewski, B., Noris, G., Sueda, S., Forberg, M., Sumner, R.W., Matusik, W., and Bickel, B. (2013). Computational design of mechanical characters. ACM Transactions on Graphics, 32(4), 1.

Folkertsma, G.A., van der Schaft, A.J., and Stramigioli, S. (2015). Morphological computation in a fast-running quadruped with elastic spine. IFAC-PapersOnLine, 48(13), 170-175.

Ha, S., Coros, S., Alspach, A., Kim, J., and Yamane, K. (2016). Task-based limb optimization for legged robots. In 2016 IEEE/RSJ International Conference on Intelligent Robots and Systems (IROS). IEEE.

He, G. and Geng, Z. (2011). Dynamics synthesis and control for a hopping robot with articulated leg. Mechanism and machine theory, 46(11), 1669-1688.

Hutter, M., Gehring, C., Lauber, A., Gunther, F., Bellicoso, C.D., Tsounis, V., Fankhauser, P., Diethelm, R., Bachmann, S., Blösch, M., et al. (2017). Anymaltoward legged robots for harsh environments. Advanced Robotics, 31(17), 918-931.

Kim, S., Wensing, P.M., et al. (2017). Design of dynamic legged robots. Foundations and Trends@ in Robotics, $5(2), 117-190$.

Lum, M.J., Rosen, J., Sinanan, M.N., and Hannaford, B. (2006). Optimization of a spherical mechanism for a minimally invasive surgical robot: theoretical and experimental approaches. IEEE Transactions on Biomedical Engineering, 53(7), 1440-1445.

Megaro, V., Zehnder, J., Bächer, M., Coros, S., Gross, M., and Thomaszewski, B. (2017). A computational design tool for compliant mechanisms. ACM Transactions on Graphics, 36(4), 1-12.

Ogura, Y., Shimomura, K., Kondo, H., Morishima, A., Okubo, T., Momoki, S., Lim, H.o., and Takanishi, A. (2006). Human-like walking with knee stretched, heelcontact and toe-off motion by a humanoid robot. In 2006 IEEE/RSJ International Conference on Intelligent Robots and Systems, 3976-3981. IEEE.

Seok, S., Wang, A., Chuah, M.Y.M., Hyun, D.J., Lee, J., Otten, D.M., Lang, J.H., and Kim, S. (2014). Design principles for energy-efficient legged locomotion and implementation on the mit cheetah robot. Ieee/asme transactions on mechatronics, 20(3), 1117-1129.

Snippe, M. (2017). Cheetah robot leg mechanism: analysis, design and cost of transport. Master's thesis, University of Twente, Netherlands.

Spröwitz, A., Tuleu, A., Vespignani, M., Ajallooeian, M., Badri, E., and Ijspeert, A.J. (2013). Towards dynamic trot gait locomotion: Design, control, and experiments with cheetah-cub, a compliant quadruped robot. The International Journal of Robotics Research, 32(8), 932950.

Xi, Y., Xia, Y., Li, X., Sun, Y., and Wang, H. (2010). Kinematic analysis and parameter optimization of sixbar linkage based on adams. In 2010 International Conference on Mechanic Automation and Control Engineering, 10-13. IEEE. 